\title{
379. Die Pioniere der Magenchirurgie im 19. Jahrhundert
}

\author{
W. Kozuschek und Ch. Hülsmann \\ Chirurgische Universitätsklinik Bochum, Knappschaftskrankenhaus Bochum-Langendreer, D-4630 Bochum
}

\section{Pioneers of Stomach Surgery in the 19th Century}

\begin{abstract}
Summary. The experiments and the first stomach resections performed in man are illustrated. The results and the original publications of the animal experiments of Merrem, Gussenbauer, Winiwarter and Wehr are presented. The second part deals with the stomach resections in humans by Péan, Rydigier and Billroth and the development of diagnostic procedures by Mikulicz with respect to stomach disease. The persons are presented, their operative techniques explained and their results demonstrated.
\end{abstract}

Key words: Stomach surgery - Mikulicz - Surgical history.

Zusammenfassung. In der Ausstellung „Die Pioniere der Magenchirurgie im 19. Jahrhundert“ werden die experimentellen Vorarbeiten und die ersten am Menschen durchgeführten Magenresektionen vorgestellt. Die Tierexperimente von Merrem, Gussenbauer und Winiwarter und Wehr sind jeweils mit ihren Ergebnissen und der Originalveröffentlichung im Bild dargestellt. Der zweite Teil der Ausstellung beschäftigt sich mit den am Menschen vorgenommenen Magenresektionen durch Péan, Rydigier und Billroth und der Weiterentwicklung der Diagnostik der Magenerkrankungen durch Mikulicz. Es werden die Personen vorgestellt, ihre Operationstechniken erläutert und ihre Ergebnisse gezeigt.

Schliisselwörter: Magenchirurgie - Mikulicz - Experimentelle Chirurgie.

\section{Gefahren durch die unipolare Diathermie für den Gefäßstiel bei Lappenplastiken}

\author{
I. Wölfl, I. Vassiliou, U. Bloch ${ }^{1}$, St. Schwindt ${ }^{1}$, K. Geißdörfer, H. Weithmann, E. Biemer ${ }^{1}$ \\ und G. Blümel \\ Institut für Experimentelle Chirurgie (Dir.: Prof. Dr. G. Blümel) und \\ ${ }^{1}$ Abteilung für Plastische Chirurgie (Vorstand: Prof. Dr. U. Schmidt-Tintemann) der Technischen Universiät \\ München, Ismaninger Straße 22, D-8000 München 80
}

\section{Dangers for the Blood Supply of Vascular Pedicle Flaps Arising from the Use of Unipolar Diathermy}

Summary. During coagulation at vascular pedicle flaps by means of unipolar diathermy the high-frequency current passes from the actual site of coagulation to the neutral electrode outside the flap, thus running through the nutrient vessels. This means that at higher amperages the nutrient vessels could suffer irreversible damage, as the current reaches its highest density there. This consideration was applied to an animal model and was fully confirmed both macroscopically and micromorphologically. These effects are not seen in bipolar diathermy.

Key words: Unipolar diathermy - Vascular pedicle flaps.

Zusammenfassung. Bei Blutstillung an gestielten Lappen mit unipolarer Diathermie fließt Strom vom Ort der Coagulation über die Gefäßstielbrücke des Lappens zur Neutralelektrode außerhalb des Lappens. Das bedeutet, bei höheren Stromstärken würden am Gefäßstiel irreversible thermische Schäden auftreten, da dieser die höchste Stromdichte aufweist. Diese Überlegung wurde am Tiermodell nachvollzogen und voll sowohl meßtechnisch als auch makroskopisch und mikromorphologisch bestätigt. Bei Anwendung der bipolaren Diathermie treten diese Effekte nicht auf, da der Strom nur zwischen den beiden Pinzettenelektroden fließt. Schlüsselwörter: Unipolare Diathermie - Gestielte Lappenplastik. 